\title{
Influence of crystal growth kinetics on prediction of macro- segregation by micro-macroscopic simulation of binary alloy solidification
}

\author{
Mirosław Seredyński ${ }^{1, *}$, and Jerzy Banaszek $^{1}$ \\ ${ }^{1}$ Institute of Heat Engineering, Faculty of Power and Aeronautical Engineering, Warsaw University of Technology, Nowowiejska 21/25, \\ 00-665 Warsaw, Poland
}

\begin{abstract}
Using the micro-macroscopic computer simulation model of binary alloy solidification, based on a single domain control-volume calculations, and involving the tracking of columnar dendrite tips envelope for distinguishing different grain structures within the two-phase liquid-solid region, the paper reports the outcomes of the detailed analysis addressing the question of the influence of enhanced KGT crystal growth models on the predicted macro-segregation pattern in the $\mathrm{Al}-4 \mathrm{wt} . \% \mathrm{Cu}$ alloy cast. In particular, the analysis concerns the impact of a proper selection of the stability constant for the KGT model (based on a crystalmelt surface energy anisotropy strength and linear scaling law of the marginal stability theory) on the predicted dendrite tip under-cooling, volumetric solid fraction and actual solute concentration along the front of columnar dendrite tips. Differences in the resulting evolution of both: the under-cooled melt region within the mushy zone and the solute concentration fields calculated for the considered various kinetics of a grain growth are also reported and discussed.
\end{abstract}

\section{Introduction}

Complex fluid flow, heat and mass transfer processes, accompanying solidification of metal alloys at the scale of individual crystals and in a whole cast, are responsible for the developing micro and macroscopic grain structures, and thus for mechanical and thermo-physical properties of a final manufactured product. Macrosegregation, i.e. a compositional heterogeneity, is a very common and serious casting defect arising due to the relative motion between the liquid and solid phases. Since this compositional heterogeneity is a large length scale phenomenon, it cannot be removed by post-casting diffusion mechanisms and homogenization heat treatment.

Tracing macro-segregation formation experimentally during the evolving process of solidification is extremely difficult; so far its ultimate picture has only been detected by post-mortem examination of the final cast. Therefore, a computationally efficient and reliable computer simulation becomes nowadays a key powerful engineering tool for designing and control of casting processes.

Such simulation involves multi-scale modelling of alloy solidification across the whole spectrum of length and time scales - including the levels of individual dendrite arms (micro-scale), a single crystal (meso-scale) and transport processes in a whole cast (macro-scale). Since a direct numerical simulation of the solidification process in its full-length and time scales for a real whole cast is still very limited due to the lack of adequate computational resources, the idea of micro-macroscopic modelling has been developed. Key calculations are performed by solving average macroscopic transport equations, and information about the developing microstructures within the two-phase region, called a mushy zone, is transferred to the macroscopic model through the effective properties of the two-phase region or/and by effective coupling of direct calculations in both these scales.

Macroscopic equations of mass, momentum, energy and species conservation can be obtained by using the classical theory of mixture (e.g. [1]) or the volume averaging (e.g. [2]). Nowadays, the most widely used formulation here is based on a fixed grid/single-domain approach and the enthalpy-porosity model, where the total enthalpy concept is applied to account for a latent heat effect, and the momentum transport within columnar mushy zone is simulated by porous medium models (e.g. [3]). Since two different dendritic structures develop within the mushy zone, i.e. columnar and equiaxed ones, it is crucial to distinguish them and then to apply different models of the momentum transport in each of them, i.e. a porous medium model or a slurry one. A common approach is based on the coherency point model [4,5], where these distinct grain structures are identified by the inspection of the solid volume fraction; when its local value exceeds some previously specified critical packing value, the region is treated as a stationary columnar mush, otherwise as an equiaxed zone. Banaszek and Seredynski [6] have contested, however, the assumption that this critical value, usually

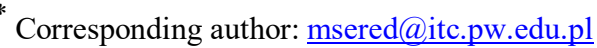


taken from experiments as equal to the solid fraction at the dendrite coherency point [5] remains constant during progressing solidification. And, in [6] they have proposed distinguishing the developing columnar and equiaxed regions through direct tracking an imaginary envelope encompassing columnar primary dendrite tips on a fixed control volume grid.

The idea of tracing the locus of columnar primary dendrite tips moving according to an assumed kinetics of crystal growth, originated by Browne and Hunt [7] for binary alloy solidification in microgravity conditions, has been further developed for alloy solidification driven by thermal and thermo-solutal natural convection to predict macro-segregation patterns on regular and nonstructural control volume grids [8,9], and for solidification in semi-transparent materials [10].

In most micro-macroscopic calculations of alloy solidification a proper choice of the crystal growth kinetics is essential. Indeed, the dendrite tip kinetics is inherent part of computer simulations based on the above-mentioned front tracking approach, on the cellular automaton coupled with the finite element method [11], in the recently developed five-phase model [12] for deducing the growth of mesoscopic envelope linking active dendrite branches, and in others.

Recently, Rebow and Browne [13] extended the most popular constrained (columnar) dendritic growth model of Kurz-Giovanola-Trivedi (KGT) [14] for aluminium alloys by new estimations of the dendrite tip stability parameter, based on measured values of their crystalmelt surface energy anisotropy strength and a simple linear scaling law of the microscopic solvability theory (MST). The influence of such an augmented 2D and 3D KGT models on the columnar mush - undercooled liquid interface position, on the solutal undercooling and the average temperature gradient at this interface, on the relative tendency to form an equiaxed zone and on the columnar to equiaxed transition (CET) phenomenon was, recently, analysed by Seredynski et al. [15] for two selected examples of $\mathrm{Al}-4 \mathrm{wt} \% \mathrm{Cu}$ alloys solidification driven only by conduction.

Considering the above, the paper presents some new results of the extended analysis involving the influence of a chosen KGT dendrite tip kinetics (with different dendrite tip stability parameters) on the predicted development of columnar and equiaxed grain regions within the mushy zone, macroscopic fields of temperature and species concentration, and thus on the numerically obtained macro-segregation patterns, for the $\mathrm{Al}-4 \mathrm{wt} \% \mathrm{Cu}$ alloy solidification driven by both molecular diffusion and thermo-solutal convection. The issue of the role of thermal and solutal buoyancy forces in the process development, when compared to a pure diffusion case, is also addressed. For these purposes, the original authors' micro-macroscopic computer simulation model is used, which is based on single domain control-volume calculations involving the tracking of columnar dendrite tips envelope (for distinguishing different grain structures within the mushy zone).

\section{Computational model}

Progress of the binary alloy solidification is described by a single set of mass, momentum, energy and solute conservation equations based on the mixture theory approach [1]:

$$
\begin{gathered}
\nabla \cdot \mathbf{V}=0 \\
\frac{\partial(\rho \mathbf{V})}{\partial t}+\nabla \cdot(\rho \mathbf{V} \mathbf{V})=\nabla \cdot\left(\mu_{l} \nabla \mathbf{V}\right) \\
-\nabla \cdot\left(\mu_{l} f_{s} \nabla \mathbf{V}_{s}\right)+\nabla \cdot\left(\mu_{s} f_{s} \nabla \mathbf{V}_{s}\right) \\
-\nabla \cdot\left[\rho \frac{f_{s}}{f_{l}}\left(\mathbf{V}-\mathbf{V}_{s}\right)\left(\mathbf{V}-\mathbf{V}_{s}\right)\right] \\
+\rho \mathbf{B}-\left(1-g_{s} \tilde{V}\right) \nabla p-\mu_{l} g_{l} \mathbf{K}^{-1} \mathbf{V} \tilde{V} \\
\frac{\partial(\rho c T)}{\partial t}+\nabla \cdot(\rho c \mathbf{V} T)=\nabla \cdot(k \nabla T) \\
+\frac{\partial\left(f_{s} \rho L_{h}\right)}{\partial t}-\nabla \cdot\left(f_{l} \rho L_{h} \mathbf{V}_{l}\right) \\
\frac{\partial(\rho C)}{\partial t}+\nabla \cdot(\rho \mathbf{V} C)=\nabla \cdot(D \nabla C) \\
+\nabla \cdot\left(f_{l} D_{l} \nabla\left(C_{l}-C\right)\right) \\
+\nabla \cdot\left(f_{s} D_{s} \nabla\left(C_{s}-C\right)\right) \\
-\nabla \cdot\left(\rho\left(\mathbf{V}-\mathbf{V}_{s}\right)\left(C_{l}-C\right)\right)
\end{gathered}
$$

where $V, p, T, C$ are, respectively, the velocity vector, pressure, temperature and solute concentration of the solid-liquid mixture. Eqs (1)-(4) are supplemented with the following assumptions and the resulting closure relations.

All material properties, except for the solutal diffusivity, are temperature independent and the same for both phases. The assumption of even phase densities implies the equality of the volumetric and mass phase fractions, i.e. $g_{i}=f_{i}$ for $i=s, l$.

Momentum equation, eq. (2), includes a special source term with the switching function, $\tilde{V}$, to mimic distinct fluid flow resistances in both porous and slurry regions developing in the mushy zone.

The volumetric force term $\rho \mathbf{B}$ of eq. (2) accounts for thermal and solutal buoyancy forces, and according to the Boussinesq's model assumption reads:

$$
\begin{aligned}
& \rho \mathbf{B}=g_{l} \rho_{l, r e f} \mathbf{g}\left[\beta_{T, l}\left(T-T_{r e f}\right)+\beta_{C, l}\left(C_{l}-C_{l, r e f}\right)\right] \\
& +g_{s} \rho_{s, r e f} \mathbf{g}\left[\beta_{T, s}\left(T-T_{r e f}\right)+\beta_{C, s}\left(C_{s}-C_{s, r e f}\right)\right]
\end{aligned}
$$

where the reference densities for the solid and liquid phases are assumed equal.

The model of micro-segregation obeys the lever rule law, so the solute concentrations in both phases are uniform and equal to $C_{l}$ and $C_{s}$, respectively. Due to distinct solute solubility in both phases, and in virtue of the equilibrium assumption, they are related by $C_{s}=$ $k_{p} C_{l}$, where $k_{p}$ stands for the partition coefficient. The 
concentration of solute in the mixture, $C$, is an average one of the two phases concentrations, and thus equal to $C=f_{s} C_{s}+f_{l} C_{l}$.

The porous zone of columnar grains is considered to be isotropic, and the Carman-Kozeny law is used: $K=\lambda_{2}^{2}\left(1-g_{s}\right)^{3} /\left(180 g_{s}^{2}\right)$, where $\lambda_{2}$ is a secondary dendrite arm spacing, taken as a time- and spatiallyindependent microstructure parameter.

The effective solid viscosity, $\mu_{s}$, is related to the effective mixture viscosity, $\mu_{m}$, with the relation, $\mu_{s}=\left[\mu_{m}-\left(1-g_{s}\right) \mu_{l}\right] / g_{s}$. The mixture viscosity is given by the commonly used rheological model, $\mu_{m}=\mu_{l}\left(1-g_{s} / g_{s, c o h}\right)^{-2.5 g_{s, c o h}}$, where $g_{s, c o h}$ is the coherency solid fraction, assumed as equal to 0.3 .

The velocity $\mathrm{V}$ is an average of the solid and liquid phase velocities $\mathbf{V}_{\mathrm{s}}$ and $\mathbf{V}_{\mathrm{l}}$, respectively, namely $\mathbf{V}=f_{l} \mathbf{V}_{l}+f_{s} \mathbf{V}_{s}$. In the slurry region, in front of the columnar dendrite tip surface, velocities of both phases are assumed to be equal, $\mathbf{V}_{1}=\mathbf{V}_{s}$. In the porous medium, velocity of the solid phase is equal to 0 . Close to the moving front of columnar dendrite tips, the phase velocities are smoothed with the switching function, $\tilde{V}$, taking $\mathbf{V}_{1}=\mathbf{V} /\left(1-f_{s} \tilde{V}\right)$ and $\mathbf{V}_{\mathrm{s}}=(1-\tilde{V}) \mathbf{V}_{1}$.

The conservation equations, eq. (1)-(4), are discretized on non-orthogonal, triangular and collocated control volume grid [16]. All variables are stored in the centres of the computational cells. The SIMPLE scheme [17] is used to solve coupled mass and momentum equations, whereas all transient terms are integrated with the fully implicit Euler scheme [17]. The second order upwind scheme is used to discretize the diffusive terms, and Rhie and Chow scheme [18] is employed to evaluate mass fluxes across the faces of control volumes.

\section{Front tracking procedure}

The envelope of columnar dendrite tips is a virtual surface, represented in the model as the loop of linear segments with mass-less markers at their ends (Fig. 1). The undercooling is determined at each marker after each time step and it is moved towards the undercooled liquid along the direction normal to the front. The actual velocity of the $i$-th marker, $\mathbf{V}_{\mathrm{t}, \mathrm{i}}$, is equal to those predicted with dendrite growth kinetics model and expressed as a function of the local undercooling, $\Delta T=T_{m}+m_{l} C-T$, where $C$ and $T$ are, respectively, the mixture concentration and the temperature interpolated at the actual marker. A new position of the marker is determined as $\mathbf{x}_{i}^{n}=\mathbf{x}_{i}^{0}+\mathbf{V}_{t, i} \Delta t$ (see [8]-[11]).

At the beginning of the next following time step, the switching function value is determined, based on the actual positions of markers (red points in Fig. 1) and the control volume grid nodes lying behind the front (green points in Fig. 1). Additionally, cross-sections of the front and the control volume faces are found (temporary markers - blue points in Fig. 1). The switching function is then calculated as the ratio of the shaded region in Fig. 1 to the volume of the actual control volume.

The proposed model has been validated by comparing its predictions with the available in the literature experimental measurements [19] and verified with the other results of computer simulation (e.g. [20]) in [9] and [15].

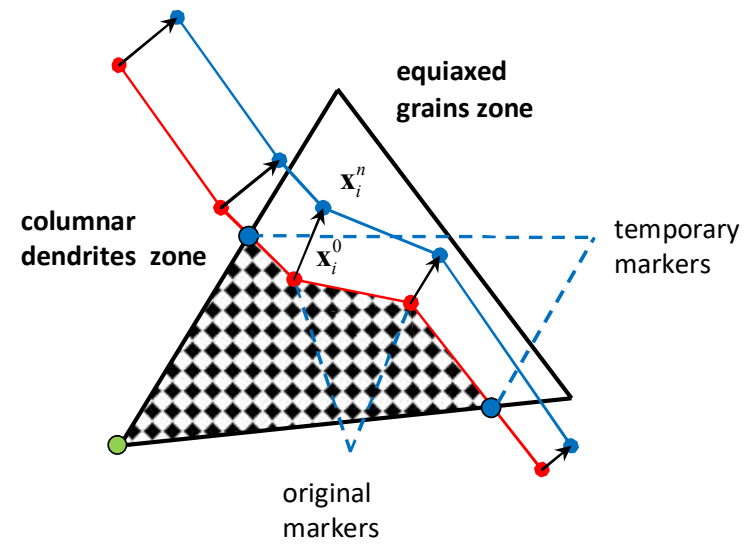

Fig. 1. Graphical representation of the front and the switching function evaluation in a control volume

A dendritic growth model is a crucial issue in micromacroscopic computer simulation of solidification because it decides on the predicted development of grain microstructure, which in turn highly influences the mechanical properties of a final casting product. The accuracy of the commonly used models of dendrite growth relies on the dendrite tip stability parameter. It is a common practice to use its constant value $\sigma^{*}=0.025$ for all alloys, which comes from the marginal stability theory (e.g. [14]). However, it was shown recently, by using 3D simulation based on the sophisticated phase field simulation [21,22] or on the theoretical analysis founded on the linear scaling law of the microscopic solvability theory [13], that this value is too small and more than twice in the case of $\mathrm{Al}-\mathrm{Cu}$ alloys. Very recently, it has been presented (see [15]) that for the Al$4 \mathrm{wt} \% \mathrm{Cu}$ alloy a chosen value of the dendrite tip stability parameter significantly influences the growth velocity, which is the function of under-cooling (Fig.2). Moreover, it also has an impact on the average temperature gradient at the columnar mush/under-cooled liquid interface, the relative tendency for forming an equiaxed zone and on the development of the columnar to equiaxed transition phenomenon. However, this analysis has been restricted to only diffusive transport, i.e. thermo-solutal convection has been neglected.

In the present paper, there is presented the extended study - involving much more challenging and real case of solidification in the terrestrial conditions, where thermal and solutal natural convection plays a critical role in the developing macroscopic heterogeneity of a species concentration. Two distinct KGT kinetics models for a columnar dendrite growth are compared. They differ in the dendrite tip stability parameter; its two different values are taken in the analysis, namely the 
commonly used one, i.e. $\sigma^{*}=0.025$, and the over twice higher proposed in [15], i.e.: $\sigma^{*}=0.058$.

The relationship between the dendrite tip velocity $V_{t}$ and the solutal tip under-cooling $\Delta T$ (shown in Fig. 2) is assumed as $V_{t}=A(\Delta T)^{n}$, where $n=3.1$ and $A\left[\mathrm{~ms}^{-1} \mathrm{~K}^{-\mathrm{n}}\right]$ takes values of $A=0.00096$ and $A=0.0022$ for $\sigma^{*}=0.025$ and $\sigma^{*}=0.058$, respectively.

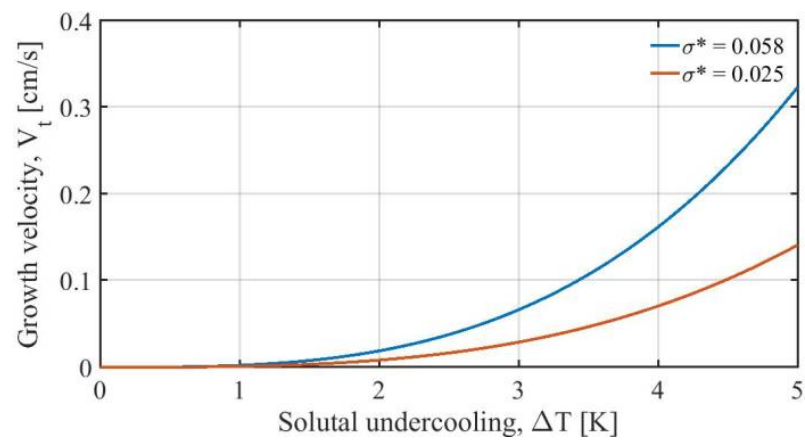

Fig. 2. Influence of the dendrite tip stability parameter on the dendrite tip velocity as a function of solutal undercooling.

Growth velocity predicted by the KGT model with $\sigma^{*}=0.058$ is more than twice higher than the one based on $\sigma^{*}=0.025$ in the considered range of undercooling (Fig.2).

\section{Problem setup}

To study the impact of the dendrite tip kinetics on the predicted formation of the undercooled slurry zone and the macro-segregation development, the above described computational model has been applied to simulate solidification of the $\mathrm{Al}-4 \mathrm{wt} \% \mathrm{Cu}$ alloy in a square casting mould of $18 \mathrm{~cm} \times 18 \mathrm{~cm}$, similar to the one used in [8]. The domain geometry, initial and boundary conditions are shown in Fig.3, whereas thermo-physical properties of the alloy are collected in Table 1.

All walls of the mould are convectively cooled with the same rate. Half of the domain (due to symmetry) is discretized with the uniform triangular grid of 8520 control volumes, and the fully implicit Euler scheme is used with time steps varying from $0.001 \mathrm{~s}$ to $0.01 \mathrm{~s}$.

\section{Results and discussion}

Solute concentration, determined with the two considered models, is compared in Fig. 4 for two different times of the cooling and solidification process, i.e. after $150 \mathrm{~s}$ and for completely solidified alloy after $450 \mathrm{~s}$.

Differences in the predicted macro-segregation pattern increase in time, but they are not pronounced. The differences of the solute concentration distribution in the completely solidified alloy are shown in Fig. 5. Positive values denote the zones, where macro-segregation calculated for $\sigma^{*}=0.025$ is higher than for $\sigma^{*}=0.058$.

Maps of the volumetric solid fraction (Fig. 6) show the similar pattern - the grains are transported with the bulk fluid downwards, and the deposit of equiaxed grains develops in the bottom of the undercooled liquid zone. In Fig.7 temporal shapes of the envelope separating the columnar mush and undercooled/equiaxed region are compared for both dendrite tip kinetics models. The difference in the velocity - undercooling relation, shown in Fig.2, makes the averaged columnar dendrite tip growth rate predicted for $\sigma^{*}=0.058$ clearly higher than for $\sigma^{*}=0.025$. So, the size of the undercooled zone, where equiaxed grains can nucleate and grow, is smaller for the former case in the whole solidification process.

Plane of symmetry.

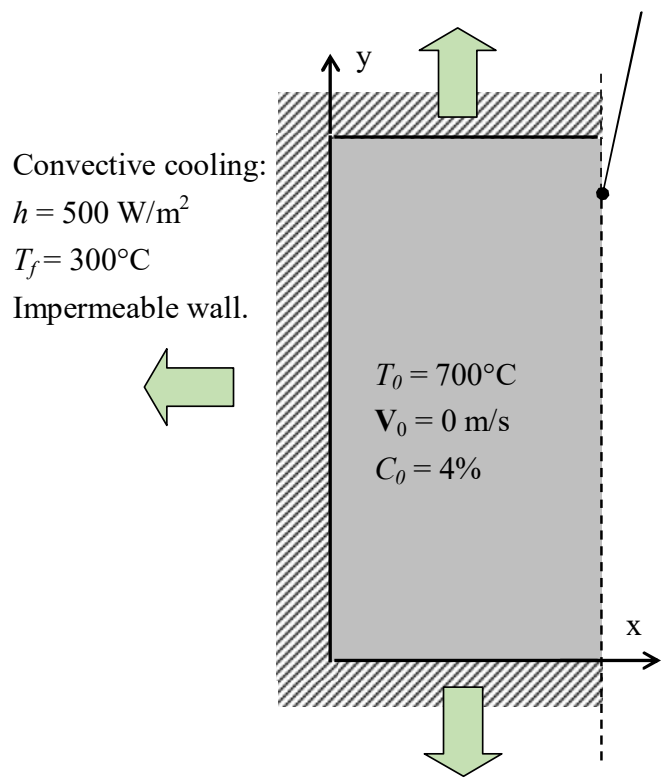

Fig. 3. Domain geometry, initial and boundary conditions for the problem analysed.

Table 1. Material properties of Al-4wt.\% $\mathrm{Cu}$

\begin{tabular}{|l|l|l|}
\hline & $\mathrm{Units}$ & Value \\
\hline Density, $\rho$ & $\mathrm{kg} / \mathrm{m}^{3}$ & 2600 \\
\hline Specific heat, $c$ & $\mathrm{~J} /(\mathrm{kgK})$ & 1000 \\
\hline Thermal conductivity, $k$ & $\mathrm{~W} /(\mathrm{mK})$ & 140 \\
\hline Latent heat of fusion, $L$ & $\mathrm{~kJ} / \mathrm{kg}$ & 390 \\
\hline Viscosity of liquid, $\mu_{l}$ & $\mathrm{~kg} /(\mathrm{ms})$ & $1.3 \cdot 10^{-3}$ \\
\hline $\begin{array}{l}\text { Thermal expansion coeff. of } \\
\text { liquid, } \beta_{T, l}\end{array}$ & $1 / \mathrm{K}$ & $1.17 \cdot 10^{-4}$ \\
\hline $\begin{array}{l}\text { Solutal expansion coeff. of } \\
\text { liquid, } \beta_{C, l}\end{array}$ & $1 /(\mathrm{wt} \%)$ & -0.0073 \\
\hline $\begin{array}{l}\text { Thermal expansion coeff. of } \\
\text { solid, } \beta_{T, s}\end{array}$ & $1 / \mathrm{K}$ & $2.25 \cdot 10^{-5}$ \\
\hline $\begin{array}{l}\text { Solutal expansion coeff. of } \\
\text { solid, } \beta_{C, s}\end{array}$ & $1 /(\mathrm{wt} \%)$ & -0.0087 \\
\hline $\begin{array}{l}\text { Melting temperature of pure } \\
\text { Al, } T_{M}\end{array}$ & ${ }^{\circ} \mathrm{C}$ & 660.35 \\
\hline Eutectic temperature, $T_{E}$ & ${ }^{\circ} \mathrm{C}$ & 580 \\
\hline Partition coefficient, $k_{p}$ & - & 0.17 \\
\hline Liquidus slope, $m l l$ & $\mathrm{~K} /(\mathrm{wt} \%)$ & -3.403 \\
\hline $\begin{array}{l}\text { Solutal diffusion coefficient in } \\
\text { liquid, } D_{l}\end{array}$ & $\mathrm{~m}^{2} / \mathrm{s}$ & $5.2 \cdot 10^{-9}$ \\
\hline $\begin{array}{l}\text { Solutal diffusion coefficient in } \\
\text { solid, } D_{s}\end{array}$ & $\mathrm{~m}^{2} / \mathrm{s}$ & $5.2 \cdot 10^{-11}$ \\
\hline Gibbs-Thompson coefficient, $\Gamma$ & $\mathrm{mK}$ & $2.41 \cdot 10^{-7}$ \\
\hline $\begin{array}{l}\text { Secondary dendrite arm } \\
\text { spacing, } \lambda_{2}\end{array}$ & $\mu \mathrm{m}$ & 100 \\
\hline
\end{tabular}


(A)

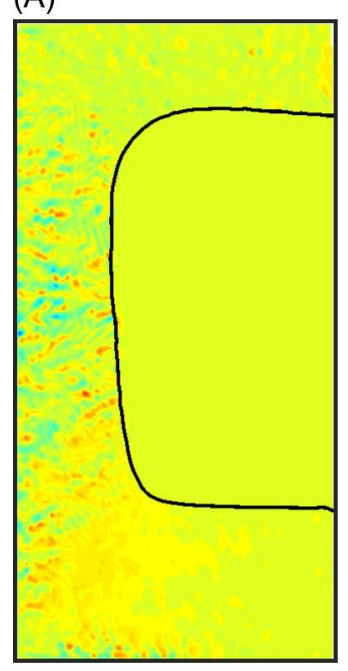

(C)

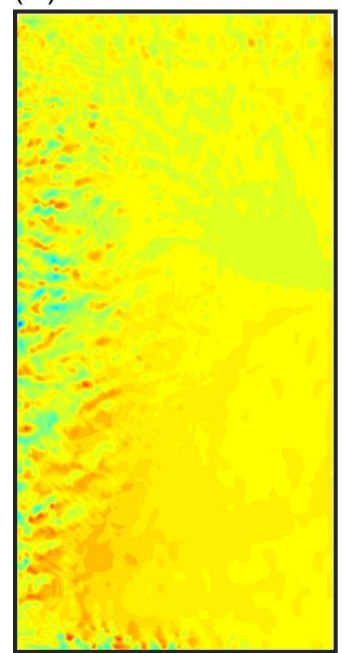

(B)

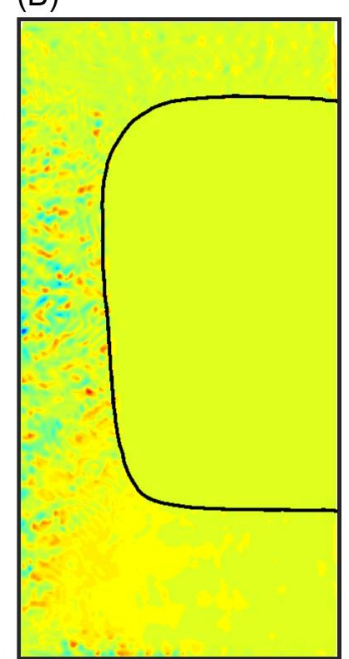

(D)

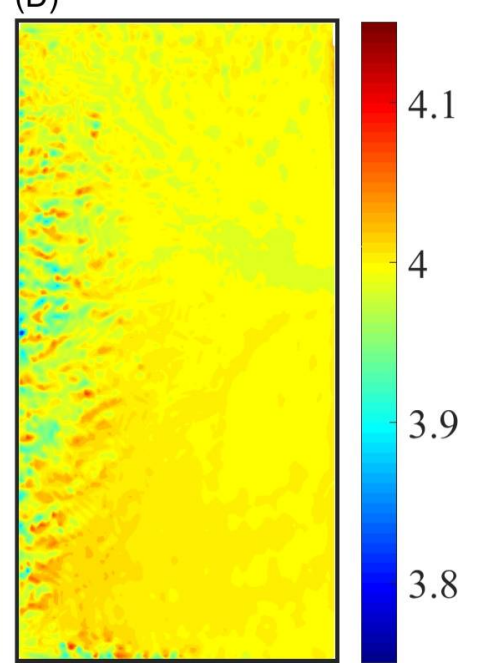

Fig. 4. Solute concentration predicted for $\sigma^{*}=0.058$ (the left column) and $\sigma^{*}=0.025$ (the right column); after $150 \mathrm{s.}$ (A and B) and $450 \mathrm{~s}$. (C and D).

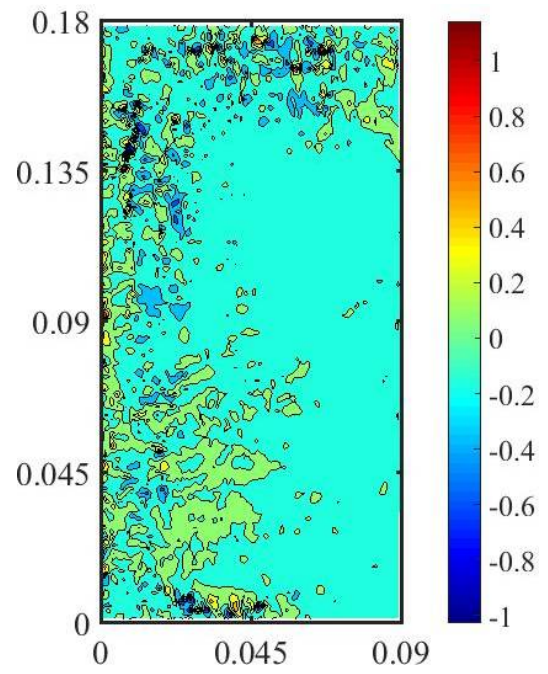

Fig. 5. Relative differences in the predicted macro-segregation patterns in the completely solidified alloy.
(A)

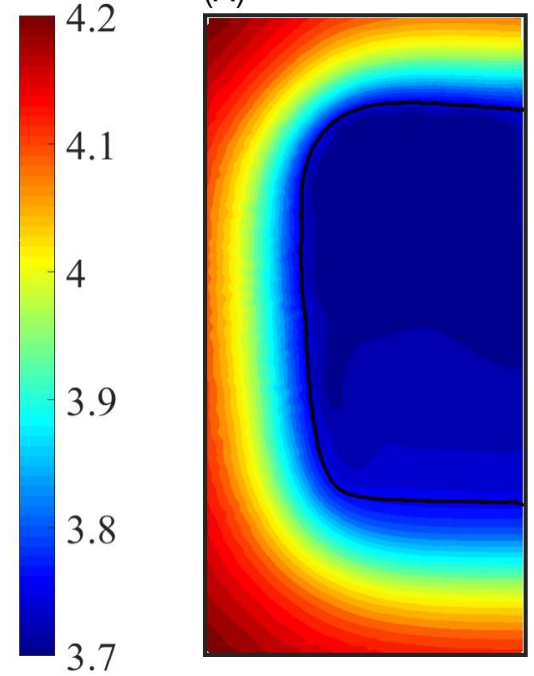

(B)

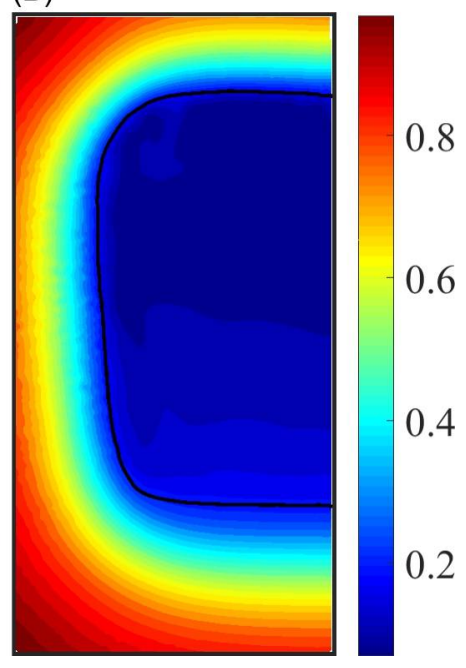

Fig. 6. Volumetric fraction of the solid phase after $150 \mathrm{~s}$. predicted for (a) $\sigma^{*}=0.058$ and (b) $\sigma^{*}=0.025$.

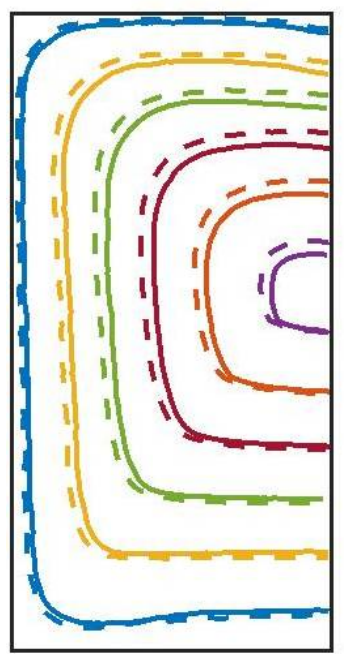

Fig. 7. Position of the front determined at different times of solidification: 50s. -blue, 100s. - yellow, 150s. - green, 200s. - marmot, $250 \mathrm{~s}$. - orange and $300 \mathrm{~s}$. - violet; a continuous line for $\sigma^{*}=0.058$ and a dashed line for $\sigma^{*}=0.025$.

Temporary variations of the averaged under-cooling along the columnar dendrite tips envelope are presented in Fig. 8. They are similar for both analysed dendrite kinetics models. After the short time period of nucleation at the cooled walls, the under-cooling predicted by both models stabilizes nearly at the same moment. Then, it slightly decreases, attains its minimum at about $100 \mathrm{~s}$ of the process, and finally slowly increases. However, the under-cooling predicted with $\sigma^{*}=0.058$ is lower in the whole analysed time. 


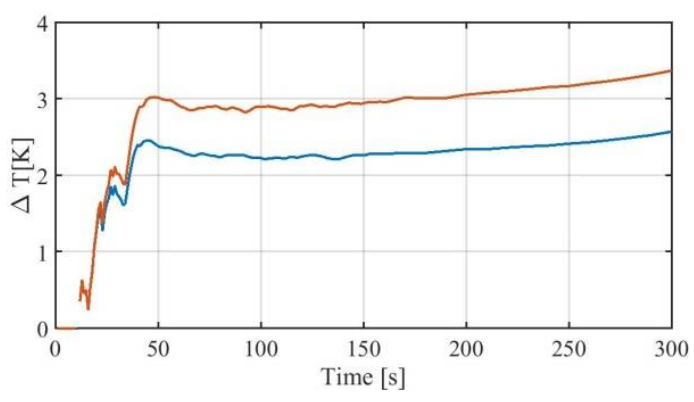

Fig. 8. Temporal changes of the averaged undercooling, for $\sigma^{*}=0.058$ - blue line, and for $\sigma^{*}=0.025$ - red line.

The predictor of the equiaxed solidification and the CET development, the equiaxed index originally proposed by Browne [23], has been further used to study the impact of the dendrite tip kinetics on the developing grain structures in the analysed $\mathrm{Al}-4 \mathrm{wt} \% \mathrm{Cu}$ solidification, in the presence of the thermo-solutal convection. This index is defined as the sum of products of local undercooling and the volume of computational cells gathered within the under-cooled zone, the domain bounded by the liquidus line and the front of columnar dendrite tips. Its time changes predicted by both the analysed dendrite tip kinetics are presented in Fig.9. The higher undercooling at the dendrite front and the larger under-cooled zone predicted for $\sigma^{*}=0.025$ result in higher values of the index, what means a better promoting the equiaxed solidification.

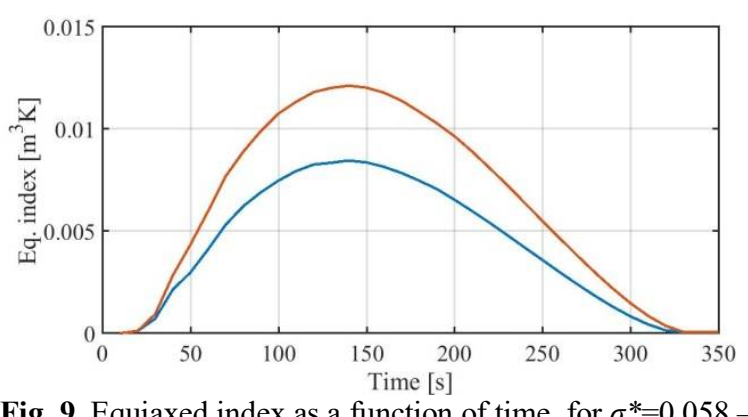

Fig. 9. Equiaxed index as a function of time, for $\sigma^{*}=0.058$ blue line, and for $\sigma^{*}=0.025$ - red line.

\section{Conclusions}

The presented analysis shows rather a weak impact of the dendrite tip stability constant in the KGT kinetics model on the numerically predicted overall macrosegregation pictures in the analysed case of the Al$4 \mathrm{wt} \% \mathrm{Cu}$ alloy solidification. One of the reasons for this may be a low mass fraction of copper in the analysed alloy, much smaller that its eutectic composition. This gives rather weak heterogeneity of the species concentration itself, comparing to alloys reach in $\mathrm{Cu}$. Moreover, in the presented calculations the equilibrium solidification was assumed and the simple model of micro-segregation, i.e. the lever-rule, used. More enhanced simulation models are needed for more precise study of the issue of the role of the dendrite tip kinetics on the accuracy of computer simulation of a multi-scale process of alloy solidification.
However, the carried out research showed visible influence of the dendrite tip kinetics on the local undercooling, temporal position of the interface between regions of different dendritic structures evolving within the mushy zone and on the potential for the development of equiaxed grain structures. Moreover, the comparison of the obtained results with those presented in [15] for $\mathrm{Al}-4 \mathrm{wt} \% \mathrm{Cu}$ solidification driven only by diffusion reveals that the presence of thermo-solutal natural convection visibly diminishes the influence of the dendrite tip kinetics model on the numerically predicted tendency for the development of equiaxed grain structures.

We are extremely grateful to Dr Marek Rebow, from the College of Engineering \& Built Environment, Dublin Institute of Technology Ireland, for sharing with us His knowledge and expertise in modelling the dendrite tip kinetics and the CET phenomenon.

\section{References}

1. J. Ni, F.P. Incropera, Int. J. Heat Mass Transfer 38, 1271 (1997)

2. J. Ni, C. Beckermann, Metall. Mater. Trans. B 22B, 349 (1991)

3. C.R. Swaminathan, V.R. Voller, Int. J. Heat Mass Transfer 40, 2859 (1997)

4. J. Ni, F.P. Incropera, Int. J. Heat Mass Transfer 38, 1285 (1995)

5. O.J. Ilegbusi, M.D. Mat, Mater. Sci. Eng., A 247A, 135 (1998)

6. J. Banaszek, M. Seredyński, Int. J. Heat Mass Transfer 55, 4334 (2012)

7. D. J. Browne, J.D. Hunt, Numer. Heat Transfer, Part B 45B, 395 (2004).

8. J. Banaszek, D.J. Browne, Mater. Trans., JIM 46, 1378 (2005)

9. M. Seredyński, J. Banaszek, J. Heat Transfer 132, 102301 (2010)

10. P. Łapka, P. Furmański, J. Heat Transfer 132, 023504 (2010)

11. Ch.-A. Gandin, J. Desbiolles, M. Rappaz, Ph. Thevoz, Metall. Mater. Trans. A 30A, 3153 (1999)

12. M. Ahmadein, M. Wu, A. Ludwig, J. Cryst. Growth 417, 65 (2015)

13. M. Rebow, D.J. Browne, Scr. Mater. 56, 481 (2017)

14. W. Kurz, B. Giovanola, R. Trivedi, Acta Metall. 34, 823 (1986)

15. M. Seredyński, M. Rebow, J. Banaszek, J. Heat Mass Transfer (2017) https://doi.org/10.1007/s00231-017-2070-z

16. H.K. Versteeg, W. Malalasekera, An introduction to computational fluid dynamics. The finite volume method (Pearson Prentice House, 2007)

17. S.V. Patankar, Numerical heat transfer and fluid flow (Mc Graw-Hill, 1980) 
18. C.M. Rhie, W.L. Chow, AIAA J. 21, 1525 (1983)

19. D.J. Hebditch, J.D. Hunt, Metall. Trans. 5, 1557 (1974)

20. P. Roux, B. Goyeau, D. Gobin, F. Fichot, M. Quinard, Int. J. Heat Mass Transfer 49, 4496 (2006)

21. K. Oguchi, T. Suzuki, Mater. Trans. JIM 48, 2280 (2007)

22. A. Choudhury, K. Reuther, E. Wesner, A. August, B. Nestler, M. Rettenmayr, Comput. Mater. Sci 55, 263 (2012)

23. D. J. Browne, ISIJ Int., 45, 37 (2005) 